\title{
Simultaneous Measurement of Optical and RF Behavior under CW and Pulsed Fully Active Harmonic Load-Pull
}

\author{
M.A. Casbon, T. Brazzini*, P.J. Tasker, M. Uren*, M. Kuball*. \\ Centre for High Frequency Engineering, School of Engineering, Cardiff University, Wales
}

Centre for Device Thermography \& Reliability, H. H. Wills Physics Laboratory, University of Bristol, UK*

\begin{abstract}
Here we present a system capable of simultaneous measurements of the RF and optical behavior of on-wafer devices, permitting the complete range of Fully Active Harmonic Load-Pull techniques to be employed while either observing optical phenomena such as Electroluminescence, or applying optical stimuli for trapping investigations. Full access to the backside of the wafer is achieved, allowing measurements on devices with source coupled field plates or air bridges, which normally obscure the gate region. Electroluminescence can be observed with an ultra-low light camera or a spectrometer. The test device was a GaN on silicon carbide HFET.
\end{abstract}

Index Terms - Waveform Engineering, fully active harmonic load-pull, power amplifiers.

\section{INTRODUCTION}

It has previously been shown that trapped charges within GaN devices can respond to light stimulation [1]-[2], and that the measurement of electroluminescence (EL) due to hot electrons is useful for reliability analysis [3]. Both of these studies are impeded by the presence of a field plate over the gate region, and source-ground air bridges, so a means of observing or stimulating the channel region from the back-side of the wafer is highly desirable. While EL analysis under RF operation was achieved by Brazzini et al, [4], this was under passive load-pull operation, which restricts the power levels and modes which can be investigated. Here we demonstrate the accurate measurement of RF I-V waveforms in a Fully Active Harmonic Load-Pull environment under pulsed or CW conditions while simultaneously performing EL microscopy or spectroscopy.

The main difference between this system and previous equipment is that the probe station was redesigned specifically to allow good access to the backside of the wafer, while not compromising the other requirements for a wafer probe station. A stable and reliable on-wafer FAHLP station was achieved, despite the range of optical facilities available.

The device used for the electroluminescence measurements was a UMS $8 \times 125 \mu \mathrm{m}$ GaN HFET on a silicon carbide substrate, the drain bias voltage was set at $28 \mathrm{~V}$.

\section{THE MEASUREMENT SysteMS}

A proven Large Signal (LSNA) system architecture based on a VTD SWAP-X402 receiver was used for this study [5]. An external modulator can be used for pulsing the RF and DC drain bias if required, with high speed RF switches to modulate the RF while a high side FET switch modulates the drain DC bias [2]. The RF and drain bias can be independently switched between pulse and $\mathrm{CW}$, enabling three different measurement conditions without making any changes to the sampling regime, therefore any measured changes can safely be ascribed to the device under test. The RF and DC switches also function as circuit breakers in the event of an overload, this permits the full range of circuit topologies such as $\mathrm{A}, \mathrm{B}, \mathrm{AB}, \mathrm{C}, \mathrm{F}, \mathrm{F}^{-1}, \mathrm{~J}$ and continuous $\mathrm{F}$ be explored in both pulse and $\mathrm{CW}$ modes, with minimal risk to the measuring equipment.

A typical wafer probe station has a large degree of movement built into all of the main features, the top-side optical microscope used for positioning, the RF wafer probes and the sample under test can all be moved, the X-Y table used for the latter normally precludes any backside access and the whole structure is complex. In fact much of this complexity is redundant, since the positioning microscope, the wafer probes and whatever instrument is accessing the backside must all address the same place within a fraction of a millimeter. By fixing the positioning microscope we can define a point of measurement which everything else can be adjusted to and then left in place, only the various devices under test need to move significantly. The concept makes it easier to align a backside fixed magnification microscope, as this can be done coarsely by removing the wafer and aligning the center of the objective with the wafer probe tips, as seen through the optical topside microscope. It is then a simple matter to finalize the alignment directly. In a similar fashion the optical fiber for the spectrometer can be aligned, in this case fine alignment is assisted by attaching an LED to the other end of the cable, in place of the spectrometer, so that the device under test appears clearly back-lit by the fiber, allowing the fiber to be centered exactly beneath the device channel. 
The station itself was made from a 19" rack frame, with a $6 \mathrm{~mm}$ steel plate attached across the top by stiffening angle sections, with cross bracing added to stiffen the frame itself. A $40 \mathrm{~mm}$ diameter hole through the center of the plate defines the measurement area. The wafer probe positioners are mounted on raised and undercut blocks, enabling any part of a 4" wafer to be placed under the probe tips. The RF measurement couplers are securely attached to the deck of the probe station, and attached to the wafer probes by short flexible cables, forming a consistent calibrated assembly. The cables to the rest of the load pull system were fed out the rear, so as to facilitate the placing of a blackout cover over the measurement area.

A custom vacuum chuck was fabricated from aluminum, to support the wafer and help to dissipate the thermal load. In addition to the usual vacuum ports to hold the wafer to the chuck and the chuck to the deck, a pattern of $5 \mathrm{~mm}$ holes through the chuck was arranged so as to permit any part of the wafer to be placed directly over a hole, by rotating the chuck relative to the wafer and deck.

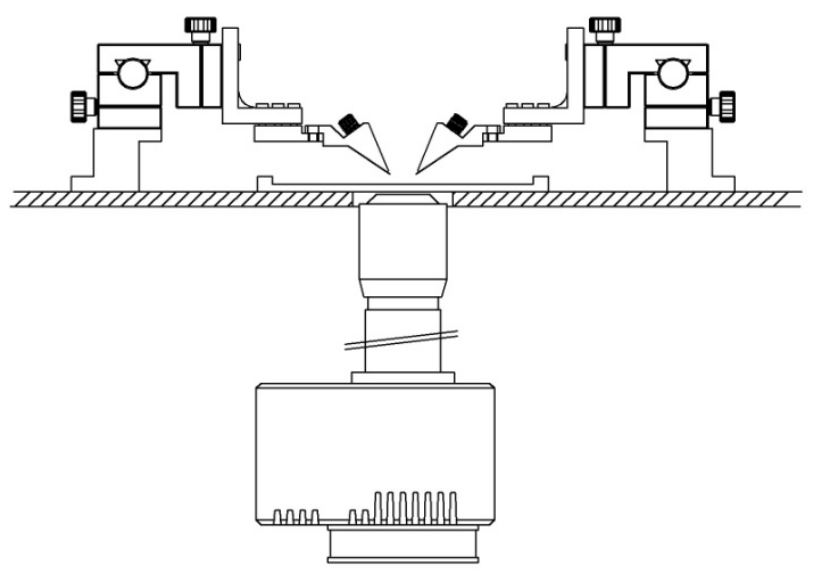

Fig. 1 The combined Optical/RF probe station.

\section{ELECTROLUMinesCENCE MEASUREMENTS}

There are two parts to the Hot Electron EL measurements, microscopy and spectroscopy, to determine intensity and spectral distribution. For the former, a Peltier cooled low light astronomical camera fitted with a x50 LWD objective lens was mounted on an XYZ micro-positioner, supported on a platform suspended beneath the deck. Initial focusing and alignment can be performed with the device powered down and with the topside light on, however since the EL is generated in the channel, beneath the device electrodes, a fine adjustment will be needed to optimize the image. Figure 2 shows a typical image with false colors. Image analysis software can be used to compare intensities in differing operational conditions. It is also possible to identify damaged areas forming "hot spots" along the channel, in case for example of OFF-state stress [6].

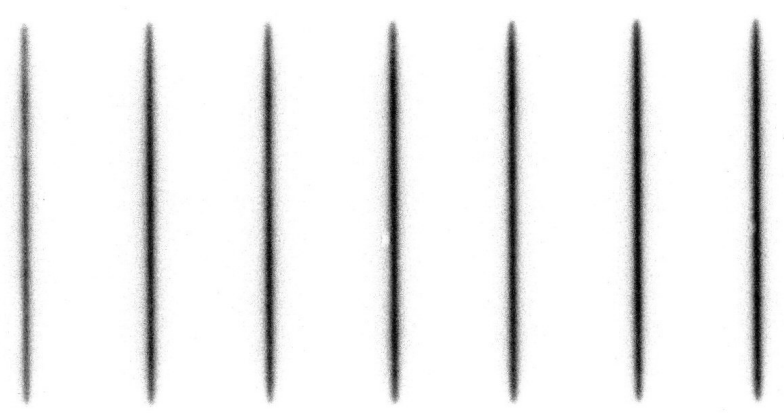

Fig. 2 The Hot Electron EL from an 8x125 GaN device, seen from the back side (monochrome, negative).

Once the intensity has been recorded for the range of conditions being studied then the microscope is replaced by the spectrometer, an Oceanoptics QEpro unit with a Peltier cooled sensor. The light is directed into the spectrometer by a broad spectrum fiber optic cable, the open end of this is attached to a micro-positioner on the underside of the probe station deck, by observing through the wafer the active area of the fiber can be centralized beneath the device under test.

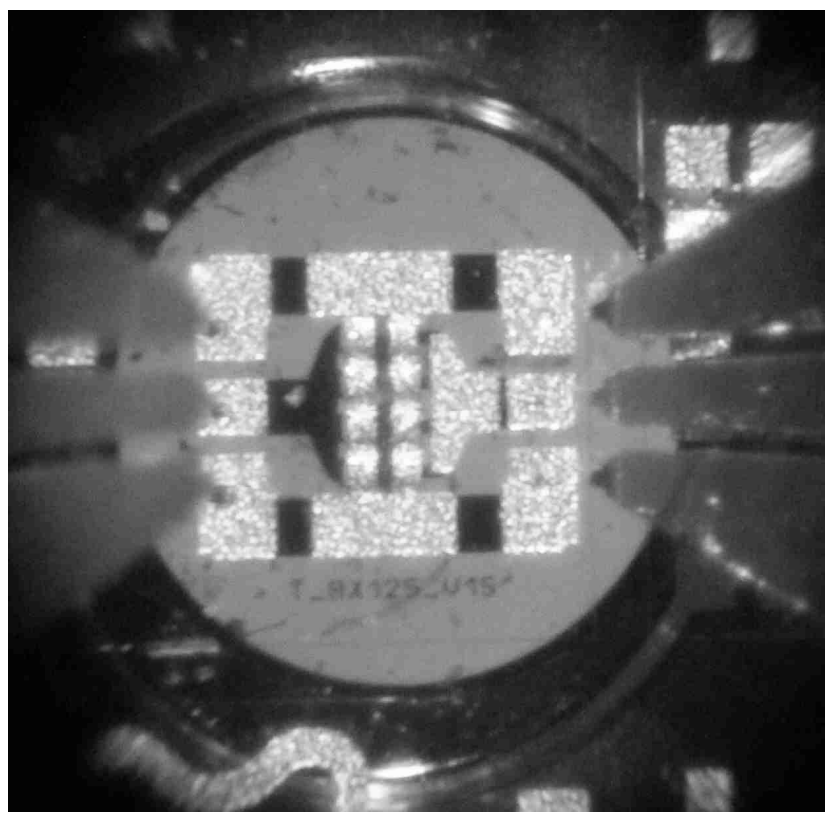

Fig. 3 The device under test backlit by the optical fiber,

Once the cable is aligned then the series of conditions measured with the camera can be repeated and the spectral distribution recorded for each case (fig. 4). After calibrating the response of the system with an Oceanoptics lamp standard, the real spectral distribution of the light emitted from the 
device can be extracted (fig. 5). The fitting of the spectra with an exponential decay form [4] can give an estimation of the electron temperature related to the carriers involved in the transport and EL emission during RF operation.

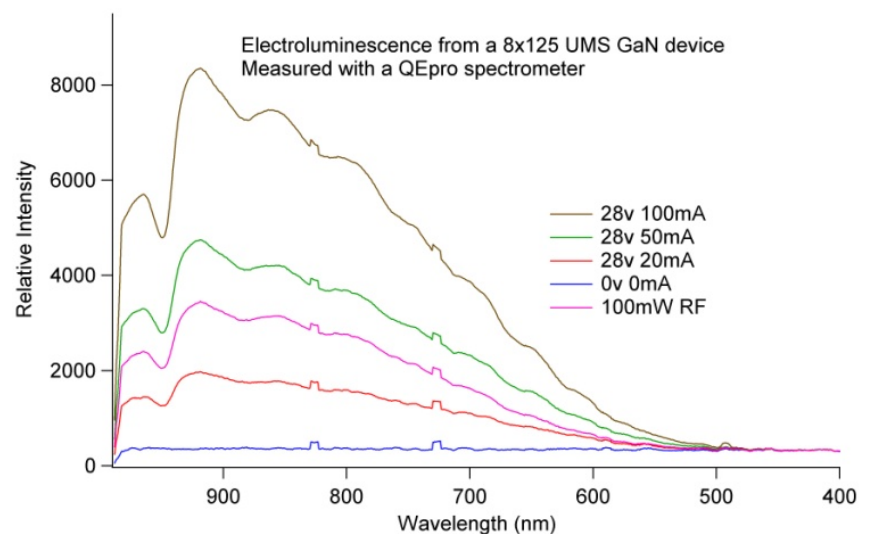

Fig. 4 Typical spectral distribution of Hot Electron EL.

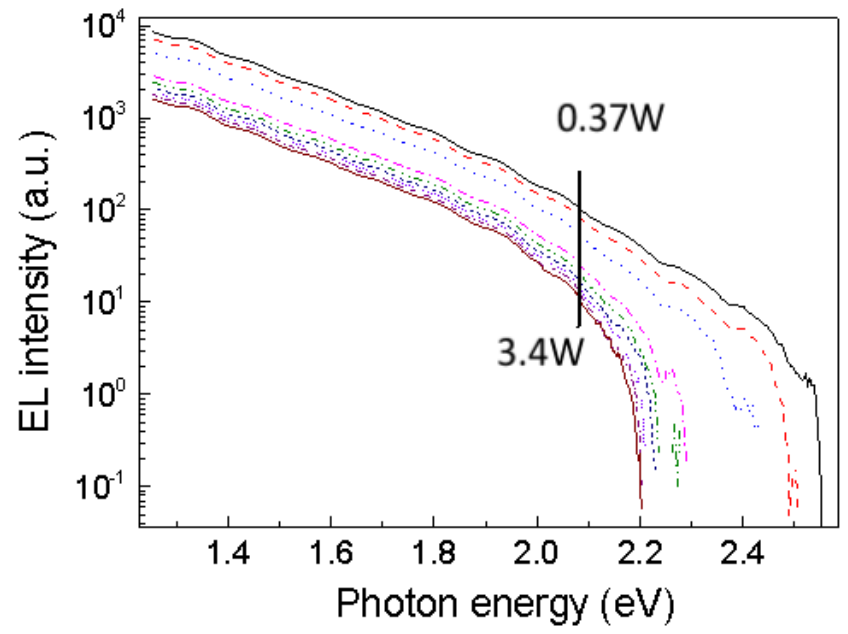

Fig. 5 Corrected distribution under Class B operation for a range of RF power levels.

As shown in the past, the EL emission analysis contains important information linked to the possible degradation due to hot electrons. Thanks to this analysis it has been pointed out how average hot electron effects in $\mathrm{AlGaN} / \mathrm{GaN}$ during RF stress may actually not have a major role when compared to a DC stress on the same load line [4]. This has been confirmed by both EL intensity and and electron temperature measurements for Class B and Class J modes of operation [7]. Highest EL intensity occurs when the current and voltage are both high, at the center of the load line. Under RF operation more time is spent near one or other of the axis while the signal sweeps over the load line, hence EL intensity is reduced as power increases (fig. 5). Instantaneous degradation effects can however take place at high voltage points during the sweeping and not be observed.

\section{Photosensitivity MEASUREMENTS}

In "Advanced RF IV Waveform Engineering Tool for use in device technology optimization: RF Pulsed Fully Active Harmonic Load Pull with Synchronized 3eV Laser" [2] the effect of short wavelength light on knee walkout in GaN devices was described, see fig. 6 , however this was purely qualitative, no attempt was made to assess the intensity of the light, the apparatus described here provides a convenient way to deliver a consistent and quantified amount of light.

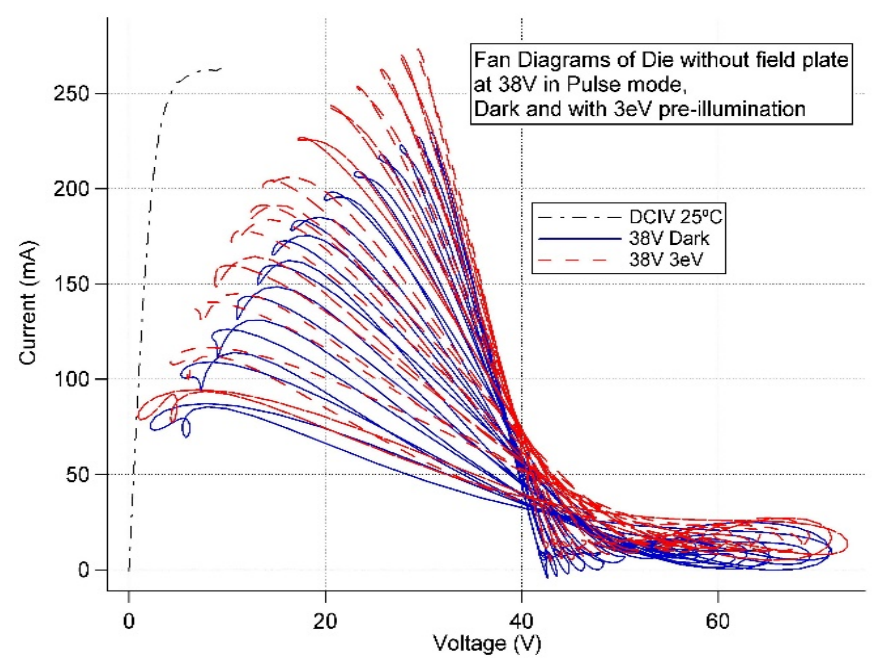

Fig. 6 The effect of $3 \mathrm{eV}(405 \mathrm{~nm})$ light on the load-lines of a $\mathrm{GaN}$ device.

In the earlier work the output from a $3 \mathrm{eV}(405 \mathrm{~nm})$ laser diode was concentrated by optics onto the top surface of the die, while effective this does not permit comparisons between light sources of different wavelengths. With this apparatus it is practical to detach the optical fiber from the probe station and connect that end to the spectrometer, so that an LED attached to the other end can be characterized, intensity vs. current. Once the cable is reattached beneath the die the effect upon the loadlines across the current range can easily be measured, this process can then be repeated for different sources. In figure 7 below we can see the effect of equal intensities of blue $(480 \mathrm{~nm})$ and violet $(395 \mathrm{~nm})$ light on the same Win Semiconductor Corp 2 x 125 devices used previously, with the fundamental, second harmonic and third harmonic loads all set to a reflection coefficient of 0.5 , and a duty cycle of $10 \%$ (it should be noted that these are prototype devices, the performance is not representative of current production parts). The blue light has a slight effect, while the violet has a pronounced effect, clearly the energy level of the photons is a significant factor in the photosensitivity. 


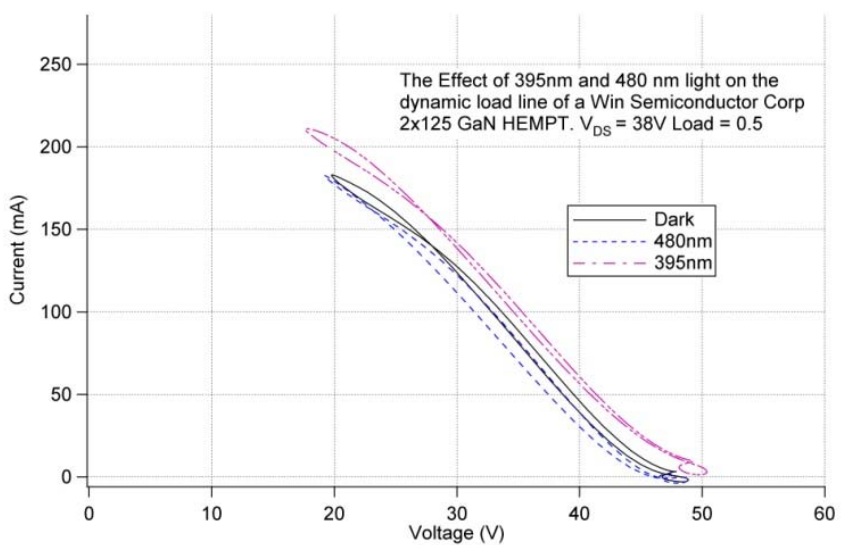

Fig. 7 The different effects of blue (480nm) and violet $(395 \mathrm{~nm})$ light on the load-line of a $2 \times 125 \mathrm{GaN}$ device.

The effect of intensity can also be observed, figure 8 shows that the rate of change of the load-line with regard to intensity is rapidly diminishing at the upper end of the experimental range, indicating that the apparatus is adequate for the task.

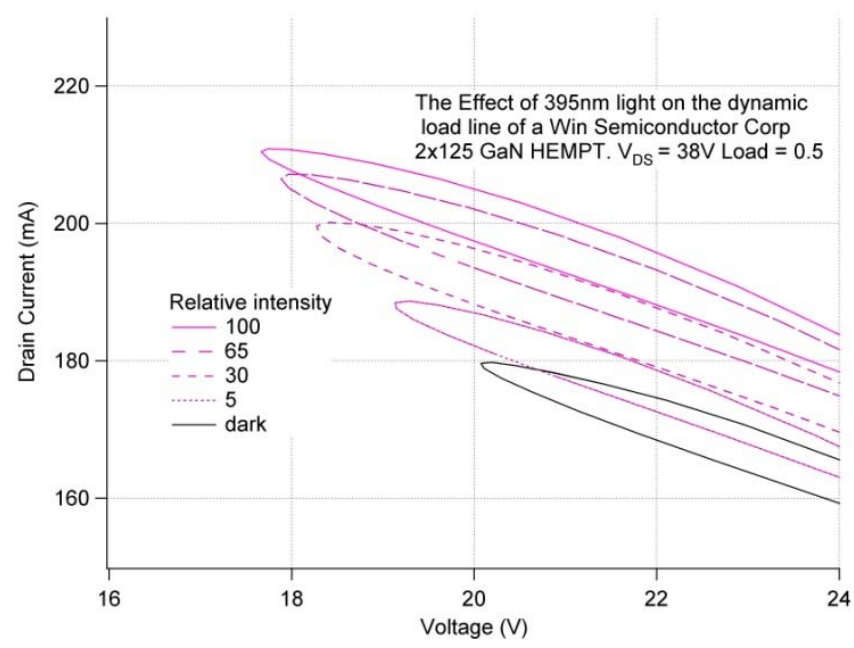

Fig. 8 The effect of violet light $(395 \mathrm{~nm})$ intensity on the load-line of a $2 \times 125 \mathrm{GaN}$ device

\section{CONCLUSION}

It has been demonstrated that accurate waveform engineering techniques can be used to comprehensively exercise an on wafer device while simultaneously measuring Hot Electron Electroluminescence, or conversely to quantify the effect of illumination on device RF behavior, and so help to diagnose process problems when developing new materials.

\section{ACKNOWLEDGEMENT}

The authors wish to acknowledge the assistance and support of EPSRC, Mesuro ltd., Win semiconductor corp. and Oceanoptics Corp. The data is included in the measurements section.

\section{REFERENCES}

[1] Youngseo Ko et al "New Thermometry and Trap Relaxation Characterization Techniques for AlGaN/GaN HEMts using Pulsed-RF Excitations" Int. Microwave Symp. Dig., Montreal, June 17-22, 2012, pp. 143-146.

[2] M.A. Casbon, P.J. Tasker, "Advanced RF IV Waveform Engineering Tool for use in device technology optimization: RF Pulsed Fully Active Harmonic Load Pull with Synchronized 3eV Laser," IEEE CSIC Symp. Dig., Monterey, CA, Oct 13-16, 2013.

.[3] M. Meneghini, A. Stocco, R. Silvestri, G. Meneghesso and E. Zanoni, "Degradation of $\mathrm{AlGaN} / \mathrm{GaN}$ high electron mobility transistors related to hot electrons" Appl. Phys. Lett. 100, $233508(2012)$

[4] T. Brazzini et al, "Electroluminescence behavior during RF operation of $\mathrm{AlGaN} / \mathrm{GaN}$ high-electron-mobility transistors", Applied Physics Letters.

[5] M. A. Casbon, P. J. Tasker and J Benedikt, "Waveform Engineering beyond the Safe Operating Region" IEEE CSIC Symp. Dig., Hawaii, HI, Oct 16-19, 2011, pp. 37-40.

[6] M. Montes Bajo, C. Hodges, M. J. Uren and M. Kuball, "On the link between electroluminescence, gate current leakage, and surface defects in $\mathrm{AlGaN} / \mathrm{GaN}$ high electron mobility transistors upon off-state stress" Appl. Phys. Lett. 101, 033508 (2012).

[7] T. Brazzini et al, "Study of hot electrons in $\mathrm{AlGaN} / \mathrm{GaN}$ HEMTs under RF Class B and Class J operation using electroluminescence", Microelectronics Reliability 55 (2015) 2493-2498 